\title{
Modeling and Designing Autonomous Control Law for Eight- Rotor UAV
}

\author{
Xiaohua Zou ${ }^{1}$ and Xiangjian Chen ${ }^{1}$ \\ ${ }^{1}$ Jiangsu University of science and technology, School of computer science and \\ Engineering, China \\ xhua.zou@163.com
}

\begin{abstract}
This paper focuses on two points: one is the details of the dynamic and kinematical model for the eight-rotor Unmanned Aerial Vehicle (UAV) which utilizes an innovative Eight-Rotor design from RCToys which has made; the other is the design of an autonomous flight control law for the Eight-Rotor Unmanned Aerial Vehicle (UAV). The approach of designing this law is decentralized in nature by incorporating the composite nonlinear feedback control, together with dynamic inversion. The overall control law consists of the core control part and command generator part. The function of the core control part is to guarantee the asymptotic stability of the Eight-Rotor UAV motion with respect to the surrounding air. The role of the command generator is to produce flight commands or references for pre-scheduled flight tasks or flight missions. Our implementation results in an efficient and robust estimation process. (C) 2001 Elsevier Science. All rights reserved.
\end{abstract}

Keywords: Autonomous Flight Control Law, dynamic and kinematical model, EightRotor UAV

\section{Introduction}

Unmanned aerial vehicles (UAVs) are remotely piloted or self-piloted aircrafts that can carry many different types of accessories. They have a very wide range of applications that include both civil and military areas. Various kinds of unmanned air vehicles have been developed for various purposes. The flight control system is a key for UAV which autonomously according to a series of pre-arranged waypoints while transmitting images of surveillance targets to control stations. The research of quad rotors [1-3] have gradually become mature in recent years, and also provided an important platform for investigations in such fields as the autonomous control, advanced sensor technology and computer science. Investigations on the control system of small scale quadrotor proved to be of high value in such applications as intelligent control of the aerial robot, 3D trajectory planning and the air traffic management and collision avoidance of multi aircrafts. However in this study, development and modelling of an Eight-Rotor helicopter were performed, the Eight-Rotor which has more advantages such as simplicity of the control system, reduced gyroscopic effects, improved stability [4-8], Damage tolerance and higher payload.

The paper begins by providing the details of the dynamic and kinematical model for the Eight-Rotor UAV which utilizes an innovative eight-rotor design from RCToys which has made Draganfly X4, Draganfly X6 and Draganfly X8. Then describe autonomous flight control law consists of two parts: the core control part, command generator part. The function of the core control part is to guarantee the asymptotic stability of the aircraft motion with respect to the surrounding air. The role of the command generator is to produce flight commands or references for pre-scheduled flight tasks or flight missions. Since the time scale associated each part of the overall flight control system is hierarchical in nature. A newly developed nonlinear control technique, namely, the 
composite nonlinear feedback (CNF) control method which has successfully been applied to solve many real-life problems, is employed to design the core control part based on the identified linear model of the UAV using in-flight data Dynamic inversion, which is capable of dealing with nonlinearities and is adopted to design the command generator based on the kinematical models of the Eight-Rotor UAV. The proposed system is evaluated by using the standard configuration of MATLAB.

\section{Dynamic and Kinematical of Eight-Rotor}

The Eight-Rotor is modelled as a rigid body using a north-east-down and a body fixed reference frame [5-9]. The flight theory of Eight-Rotor will be introduced as follows: eight rotors are installed in the four vertex Positions of the body. The four pairs of propellers as described in Figure1. Turn in opposite directions. By varying the rotor speed, one can change the lift force and create motion. Thus, increasing or decreasing the eight propeller's speeds together generates vertical motion. It will hover in the air when there is no net force in any degree of freedom and force balance for a stable hover is achieved when the sum of the thrust from eight rotors equals the weight of the craft.

Considering earth fixed frame $E=(x, y, z)$ and body fixed frame $B=\left(x_{b}, y_{b}, z_{b}\right)$ as seen in Figure1. Formatting the equation expressed as Eq. (1) between the forces and the moments in the body fixed frame, the equation was based on the Newton-Euler formalism.

$$
\left\{\begin{array}{l}
m \ddot{u}+(q w-v r) m=\Sigma F_{x b} \\
m \dot{v}+(r u-p w) m=\Sigma F_{y b} \\
m \dot{w}+(p v-u q) m=\Sigma F_{z b} \\
I_{x} \dot{p}+\left(I_{z}-I_{y}\right) q r=\Sigma M_{x b} \\
I_{y} \dot{p}+\left(I_{z}-I_{z}\right) q r=\Sigma M_{y b} \\
I_{z} \dot{p}+\left(I_{y}-I_{x}\right) q r=\Sigma M_{z b}
\end{array}\right.
$$

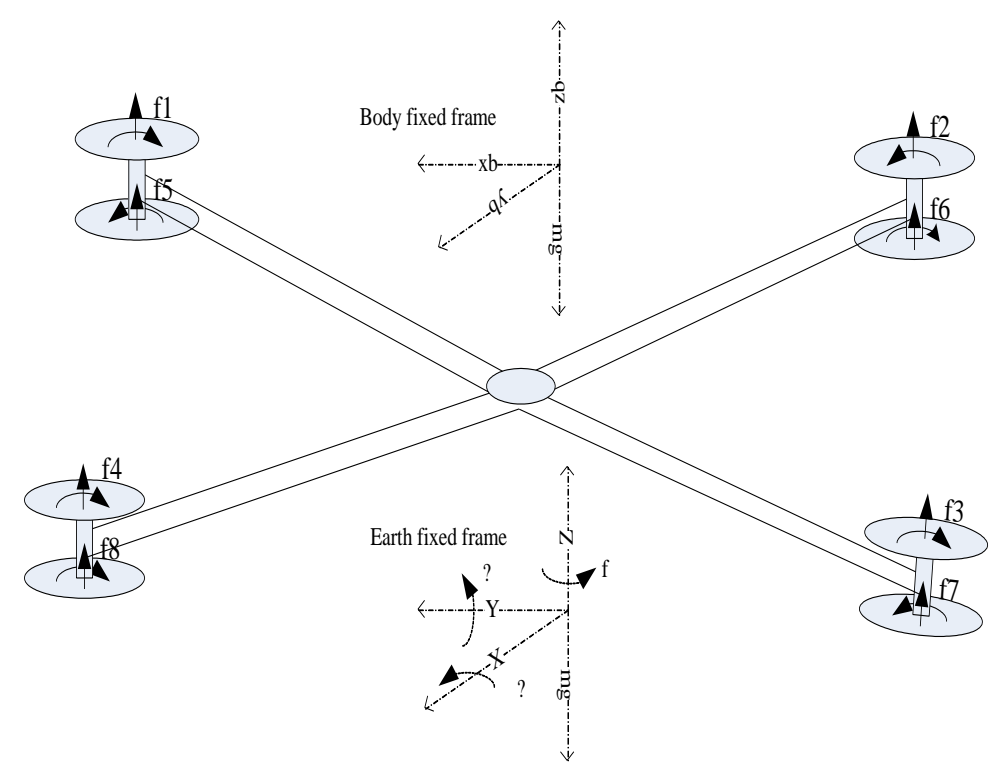

Figure 1. Dynamic Modelling for Eight-Rotor UAV

Velocities are described in a body fixed frame with linear velocity $(\mathrm{u}, \mathrm{v}, \mathrm{w})$. The velocity u points from aft to fore, $v$ to starboard, and w from top to bottom. The vector ( $p$, 
$\mathrm{q}, \mathrm{r}$ ) is the angular velocity of the body fixed frame relative to the NED frame, decomposed in the body fixed frame.

$\left(\Sigma F_{x b}, \Sigma F_{y b}, \Sigma F_{z b}\right)$ means total external forces in the body fixed frame. $\left(\Sigma M_{x b}, \Sigma M_{y b}, \Sigma M_{z b}\right)$ means total external torques in the body fixed frame. $\left(I_{x}, I_{y}, I_{z}\right)$ is axial moment of inertia. The quality of the Eight-Rotor is $\mathrm{m}$. Approximating that EightRotor is symmetrical relative to the $x o z$ and the ${ }^{y o z}$. Therefore, it can be assumed that $\left(I_{x y}, I_{y z}, I_{x z}\right)$ is zero.

Velocity (u, v, w) in the body fixed frame expressed as Eq. (2) by using R inertia matrix:

$$
\left[\begin{array}{c}
\dot{x} \\
\dot{y} \\
\dot{z}
\end{array}\right]=\left[\begin{array}{ccc}
\cos \psi \cos \theta & \cos \psi \sin \theta \sin \phi-\sin \psi \cos \phi & \cos \psi \sin \theta \cos \phi+\sin \psi \sin \theta \\
\sin \psi \cos \theta & \sin \psi \sin \theta \sin \phi+\cos \psi \cos \theta & \sin \psi \sin \theta \cos \phi-\sin \phi \cos \psi \\
-\sin \theta & \cos \theta \sin \phi & \cos \theta \cos \phi
\end{array}\right] *\left[\begin{array}{l}
u \\
v \\
r
\end{array}\right]
$$

The lift force of the Eight-Rotor is expressed as (3) in the body fixed frame:

$$
F_{v t}=\sum_{i=1}^{8} T_{b i} \bar{z}_{b}=k_{1}\left(\Omega_{1}^{2}+\Omega_{2}^{2}+\Omega_{3}^{2}+\Omega_{4}^{2}+\Omega_{5}^{2}+\Omega_{6}^{2}+\Omega_{7}^{2}+\Omega_{8}^{2}\right)
$$

The torque applied on the vehicle's body along an axis is the difference between the torques generated by each propeller on the other axis.

$$
\left\{\begin{array}{l}
M_{1}=l k_{1}\left(\Omega_{4}^{2}+\Omega_{6}^{2}-\Omega_{2}^{2}-\Omega_{8}^{2}\right) \\
M_{2}=l k_{1}\left(\Omega_{3}^{2}+\Omega_{5}^{2}-\Omega_{1}^{2}-\Omega_{7}^{2}\right) \\
M_{3}=l d\left(\Omega_{4}^{2}+\Omega_{2}^{2}+\Omega_{5}^{2}+\Omega_{7}^{2}-\Omega_{3}^{2}-\Omega_{1}^{2}-\Omega_{6}^{2}-\Omega_{8}^{2}\right)
\end{array}\right.
$$

The full Eight-Rotor UAV dynamic model with the $x, y, z$ motions as a consequence of a pitch or roll rotation, the model has shown in Eq. (5) and (6).

$$
\begin{aligned}
& \left\{\begin{array}{l}
\ddot{x}=\frac{R \sum F_{x b}}{m}=\frac{(\sin \theta \cos \phi \cos \psi+\sin \psi \sin \phi) U_{1}}{m} \\
\ddot{y}=\frac{R \sum F_{y b}}{m}=\frac{(\sin \theta \cos \phi \sin \psi-\cos \psi \sin \phi) U_{1}}{m} \\
\ddot{z}=\frac{R \sum F_{z b}}{m}=\frac{(\cos \theta \cos \psi) U_{1}}{m}-g
\end{array}\right. \\
& \left\{\begin{array}{l}
\ddot{\phi}=\frac{\sum M_{x}-\dot{\theta} \dot{\psi}\left(I_{z}-I_{y}\right)}{I_{x}}=\frac{U_{2} l+\dot{\theta} \dot{\psi}\left(I_{z}-I_{y}\right)-J_{r} \dot{\theta} \Omega}{I_{x}} \\
\ddot{\theta}=\frac{\sum M_{y}-\dot{\phi} \dot{\psi}\left(I_{x}-I_{z}\right)}{I_{y}}=\frac{U_{3} l+\dot{\phi} \dot{\psi}\left(I_{x}-I_{z}\right)-J_{r} \dot{\phi} \Omega}{I_{y}} \\
\ddot{\psi}=\frac{\sum M_{z}-\dot{\phi} \dot{\theta}\left(I_{y}-I_{z}\right)}{I_{z}}=\frac{U_{4} l+\dot{\phi} \dot{\theta}\left(I_{y}-I_{z}\right)}{I_{z}}
\end{array}\right.
\end{aligned}
$$

Then, the system's inputs are posed $U_{1}, U_{2}, U_{3}, U_{4}$ and $\Omega$ obtained as shown in Eq. (7)

$$
\left\{\begin{array}{l}
U_{2}=k_{1}\left(\Omega_{4}^{2}+\Omega_{6}^{2}-\Omega_{2}^{2}-\Omega_{8}^{2}\right) \\
U_{3}=k_{1}\left(\Omega_{3}^{2}+\Omega_{5}^{2}-\Omega_{1}^{2}-\Omega_{7}^{2}\right) \\
U_{4}=k_{d}\left(\Omega_{4}^{2}+\Omega_{2}^{2}+\Omega_{5}^{2}+\Omega_{7}^{2}-\Omega_{3}^{2}-\Omega_{1}^{2}-\Omega_{6}^{2}-\Omega_{8}^{2}\right) \\
\Omega=\Omega_{2}+\Omega_{4}+\Omega_{5}+\Omega_{7}-\Omega_{1}-\Omega_{3}-\Omega_{6}-\Omega_{8}
\end{array}\right.
$$

The dynamic equations of Eight-Rotor have been finished: $(x, y, z)$ is the location coordinate of the centroid for the Eight-Rotor, $(\phi, \theta, \psi)$ is the pitch angle, roll angle and yaw angle; $g$ is the acceleration of gravity; $\left({ }_{x}, I_{y}, I_{z}\right)$ is the inertia of the main axial.. 


\section{Eight-Rotor UAV System Architecture}

\subsection{Introduction for Draganfly X8}

The Eight-Rotor shown in Figure2 utilizes an innovative eight-rotor design from RCToys. The eight rotors are arranged as four counter-rotating offset pairs mounted at the ends of the four arms, with matched sets of counter-rotating rotor blades. Differential thrust from these four equally spaced points make the Eight-Rotor able to maneuver quickly and precisely. The offset layout doubles the thrust without increasing the size of the footprint, and naturally eliminates loss of efficiency due to torque compensation. The rotors are optimized for maximum efficiency, low noise, low weight, and high strength.

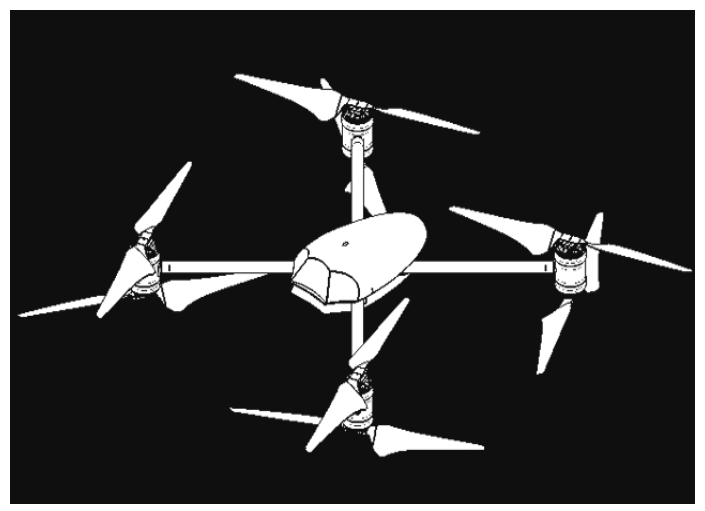

Figure 2. Draganfly Eight-Rotor (X8)

The rotors are mounted on brushless motors designed to direct drive them with maximum power and efficiency. The motors are mounted to the four-arm carbon fibbers frame designed for maximum strength and minimum weight. Eight efficient, electric motors allow the Eight-Rotor to carry heavier and more advanced payloads while providing more safety features than ever before.

\subsection{Hardware Design for Eight-Rotor UAV}

The hardware architecture for the Eight-Rotor autonomous flight control system shown in Figure 3 includes sensor modules, microprocessor unit, wireless transmission module, payload module, throttle module, and power management module, and Servo Control Module.

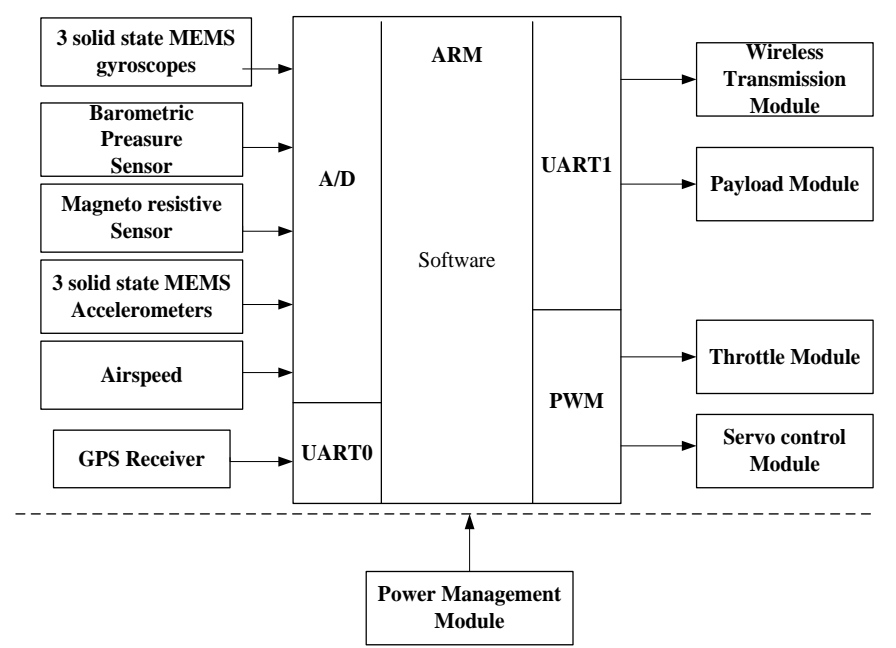

Figure 3. Architecture of Hardware Design 
In the sensor modules, the data from the 3 solid state MEMS gyroscopes and 3 solid state MEMS Accelerometers is used to stabilize the UAV, the Magneto resistive sensor measures the course, the Barometric pressure sensor measures the airspeed, and the GPS was used for position-hold, location and velocity data.

The microprocessor unit used S3C2410A which offers outstanding features with its CPU core, a 16/32-bit ARM920T RISC processor designed by Advanced RISC Machines. The ARM920T implements MMU, AMBA BUS, and Harvard cache architecture with separate $16 \mathrm{~KB}$ instruction and $16 \mathrm{~KB}$ data caches, each with an 8 -word line length. By providing a complete set of common system peripherals, the S3C2410A minimizes overall system costs and eliminates the need to configure additional components. The integrated on-chip functions that are described: $1.8 \mathrm{~V} / 2.0 \mathrm{Vint}$, microprocessor with $16 \mathrm{~KB}$ I-Cache,16KB Cache MMU; External memory controller (SDRAM Control and Chip Select logic); 4-ch DMAS with external request pins; 3-ch UART, 2-ch SPI; SD Host interface version $1.0 \&$ Multi-Media Card Protocol version 2.11 compatible; 2-port USB Host /1- port USB Device; 4-ch PWM timers \& 1-ch internal timer;117-bit general purpose I/O ports / 24-ch external interrupt source; 8-ch 10-bit ADC and Touch screen interface; On-chip clock generator with PLL

\section{Autonomous Flight Control Law Design}

The block diagram of the autonomous flight control law [10-15] is shown in Figure4, in which the overall law is hierarchically divided into two parts: the core control law, which is to guarantee the asymptotic stability of the aircraft motion with respect to the surrounding air and to track flight commands; the command generator law, which is to generate flight commands by tracking flight scheduling references based on pre-schedule flight tasks or flight missions. As demonstrated later in simulation results, such a control scheme has proven to yield an excellent performance.

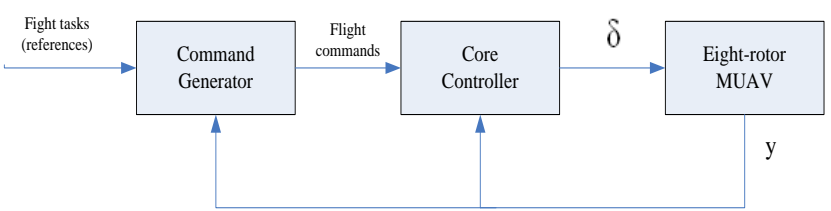

Figure 4. Structure of the Autonomous Flight Control Law

\subsection{Core Control Law}

The core control law is decoupled into two parts: the longitudinal control, lateral control and heaving control and heading control components. The longitudinal, lateral and directional controllers are designed with proportional-integral (PI) method, whereas the heading control laws are designed using the CNF control technique. The CNF controller consists of a linear feedback control law and a nonlinear feedback control law. The linear feedback law is designed to yield a closed-loop system with a small damping ratio for a quick response, while the nonlinear feedback law is used to increase the damping ratio of the closed-loop system.

\subsubsection{Longitudinal Control System}

Pitch rate feedback was employed to add artificial pitch damping to the Eight-Rotor UAV and quickly negate the adverse effects of disturbance torques. Given that disturbance torques were likely to arise from low-frequency phenomena such as offsets in the thrust action line and constant winds, a proportional-integral (PI) type controller was used. This architecture allowed for both high bandwidth pitch rate feedback and fast rejection of steady state disturbance torques. The final control law was thus of the form: 


$$
\delta_{p}=k_{L_{P}}\left(L_{I}-L\right)+k_{L_{t}} \int_{t}\left(L_{I}-L\right) d t
$$

Where $\mathrm{R}$ denotes a reference variable. With the pitch rate controller in place, a pitch angle controller was designed to regulate the aircraft's longitudinal attitude. With the pitch angle controller driving into the pitch rate controller, pure proportional control leaves the pitch angle controller susceptible to biases in the pitch rate measurement/estimate. It was decided that implementing more complex controller to handle the bias was not worthwhile. Thus, the final pitch angle controller was of the form:

$$
L_{I}=K_{\theta}\left(\theta_{L}-\theta\right)
$$

An integrator would serve to compensate for any steady state forces that may act on the system such as those due to constant winds. The longitudinal velocity controller was thus of the form:

$$
\theta_{L}=K_{U}\left(U_{L}-U\right)
$$

\subsubsection{Lateral Control}

Due to the symmetry of the eight-rotor MUAV about the duct centre line, the lateral dynamics are of exactly the same form as the longitudinal dynamics. Thus, the architecture of the lateral control system was made identical to the longitudinal control system. For that reason, three control laws are stated below:

$$
\begin{gathered}
\delta_{R}=k_{I_{P}}\left(L_{L}-L\right)+k_{I_{t}} \int_{t}\left(I_{L}-I\right) d t \\
I_{L}=K_{\phi}\left(\phi_{L}-\phi\right) \\
\theta_{L}=K_{V}\left(V_{L}-V\right)
\end{gathered}
$$

\subsubsection{Heave Motion Control}

With reference to the heave dynamics, an inner-loop sink rate controller was designed first. The primary purpose of this PI controller was to provide damping of the heave dynamics and to quickly negate steady state disturbances due to factors such as trim thrust variations. The control law was thus:

$$
\delta_{T R}=k_{W_{I}}\left(W_{L}-W\right)+k_{W_{t}} \int_{t}\left(W_{L}-W\right) d t
$$

A proportional feedback altitude controller was then implemented in the knowledge that steady state disturbances would be removed by the inner sink rate controller. The control law was thus:

$$
W_{L}=K_{D}\left(D_{L}-D\right)
$$

\subsubsection{Heading Motion Control}

Heading motion control: Heading motion control is to generate a controller such that the state variable will follow the flight command. The subsystem we use for heading action control is characterized. By following state feedback CNF control law yields a very good performance for the heading motion:

$$
V_{h}=\left[\begin{array}{ll}
0.02 & -0.08
\end{array}\right]\left(\begin{array}{c}
\phi \\
\omega_{z}
\end{array}\right)+K_{G \phi}+\rho_{\phi}[0-58.42]\left(\left(\begin{array}{c}
\phi \\
\omega_{z}
\end{array}\right)-K_{H \phi} \phi_{c}\right)
$$

Where the conditions for the solution of the Lyapunov equation is:

$$
K_{G \varphi}=-\left[\left(\begin{array}{ll}
1 & 0
\end{array}\right)\left(\begin{array}{cc}
0 & 1 \\
0 & -6
\end{array}\right)+\left(\begin{array}{ll}
0 & -58.42
\end{array}\right)\left(\begin{array}{c}
0.02 \\
-0.08
\end{array}\right)\right]^{-1}\left[\begin{array}{c}
0 \\
-58.42
\end{array}\right]^{-1}
$$




$$
\begin{gathered}
K_{H \varphi}=-\left[\left[\begin{array}{cc}
0 & 1 \\
0 & -5.5
\end{array}\right]+\left(\begin{array}{c}
0 \\
-58.42
\end{array}\right)\left(\begin{array}{ll}
0.02 & -0.08)
\end{array}\right]^{-1}\left(\begin{array}{c}
0 \\
-58.42
\end{array}\right) K_{G \varphi}\right. \\
\rho_{\varphi}=\left|\frac{\boldsymbol{e}^{\varphi-\varphi} \boldsymbol{c} \mid-\boldsymbol{e}^{-1}}{1-\boldsymbol{e}^{-1}}\right|<0
\end{gathered}
$$

While the actual control signal that is injected into the UAV is given by:

$$
\delta=\delta_{0}+u
$$

\subsection{Command Generator Law}

The command generator function is to generate necessary flight commands associated with required flight missions. It can be carried out using the dynamic inversion technique based on the displacement equation, we note that:

$$
\left[\begin{array}{c}
\dot{x} \\
\dot{y} \\
\dot{z}
\end{array}\right]=R^{\prime} * V_{g}\left[\begin{array}{c}
\cos \varphi_{s} \cos \theta_{s} \\
\sin \varphi_{s} \cos \theta_{s} \\
-\sin \theta_{s}
\end{array}\right]
$$

Where $V_{g}, \varphi_{s}, \theta_{s}$ are respectively the ground speed, flight path angle and flight azimuth angle. The task of the command generator is to generate flight commands. For example: $U_{c}, V_{c}, R_{c}, \varphi_{c}$ by tracking the flight references of the scheduled steady and maneuvering flights. For a heading direction reference given in terms of $\varphi_{c}=\varphi_{r}$. For flight references given in terms of $X_{r}, Y_{r}, Z_{r}$ or $h_{r}$.

$$
\left[\begin{array}{c}
\dot{x} \\
\dot{y} \\
\dot{z}
\end{array}\right]=R^{\prime} *\left[\begin{array}{c}
K_{1 x}\left(x-x_{r}\right) \\
K_{2 x}\left(y-y_{r}\right) \\
K_{3 x}\left(z-z_{r}\right)
\end{array}\right]
$$

If $h_{r}=-z$, then given:

$K_{1 x}=-0.2, K_{2 x}=-0.3, K_{3 x}=-0.6$ are feedback gains chosen for our Eight-Rotor UAV. For flight references given in terms of $V_{g r}$ and either one of $\theta_{r_{\text {and }}} \varphi_{r}$ :

$$
\begin{gathered}
{\left[\begin{array}{c}
\dot{x} \\
\dot{y} \\
\dot{z}
\end{array}\right]=R^{\prime} * V_{g r}\left[\begin{array}{c}
\cos \varphi_{c} \cos \theta_{c} \\
\sin \varphi_{c} \cos \theta_{c} \\
-\sin \theta_{c}
\end{array}\right]} \\
\theta_{c}=\arcsin \left\{\frac{k(h+Z)}{V_{g}}\right\}, \varphi_{c}=\varphi_{r}
\end{gathered}
$$

Where $\mathrm{k}=-0.5$ is a feedback gain chosen for Eight-Rotor UAV

\section{Simulation}

We used our own built hardware-in-the-loop simulation system on which we run a simulation test. Flight scheduling consists of tasks including automatic takeoff, hovering, spiral turning, heading turn and automatic landing. Lately gives the event-driven models of such an experiment. The flight references of the scheduled steady and maneuvering flights are given as follows: Takeoff: Transition condition Lift up $25 \mathrm{~m} ;{ }_{r}, Y_{r}, h_{r}$ and $\varphi_{r}$ are constants; 
Hovering: Transition condition-20s for duration; $X_{r}, Y_{r}, h_{r}$ and $\varphi_{r}$ are constants;

Slithering: Transition condition is $32 \mathrm{~s}$ for duration; $\varphi_{r}=\varphi_{r 0} \pm \pi / 3, h_{r}, V_{g r}, \varphi_{r}$ are constants;

Heading turn: Transition condition is 32 s for duration; $h_{r}, V_{g r}, \varphi_{r}$ are constants;

Pirouetting: Transition condition-descend to ground; $X_{r}, Y_{r}, h_{r}$ and $\varphi_{r}$ are constants. Shown in Figure5 and Figure6 are the comparison of the performance of the flight control laws designed using the CNF control technique and that of their linear counterparts for dynamical motion. It is clear that the former outperform the linear ones.

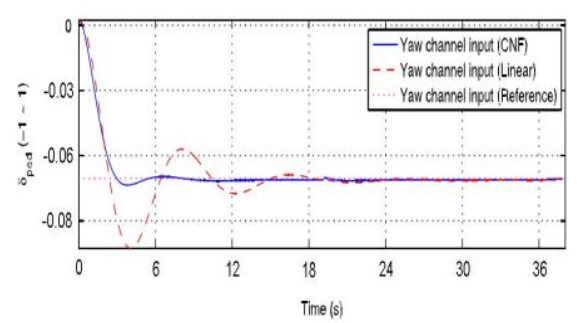

Figure 5. Heading and Positioning Responses for Pirouetting Motion

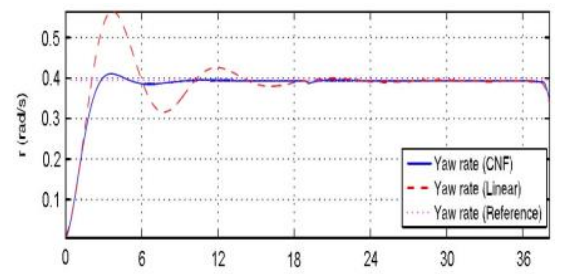

Figure 6. Yaw Rate Responses for Pirouetting Motion

\section{Conclusion}

The autonomous flight control law has been designed for our Eight-Rotor UAV with a decentralized scheme incorporating the composite nonlinear control technique and the dynamic inversion approach. The design has not been successfully verified in the actual flight tests. The result of closed-loop system shows that our design has achieved top level flight performance in our simulation.

\section{Acknowledgments}

This paper is supported by National Natural Science Found of China No.61502211.

\section{References}

[1] E. Altug, J. P. Ostrowski, and R. E. Mahony, "Control of a Quadrotor Helicopter using Visual Feedback", ICRA, (2002), pp. $72-77$.

[2] P. Mckerrow, "Modeling the draganflyer four-rotor helicopter", In Proceedings of the 2004 IEEE international conference on robotics and automation, (2004), pp. 3596-3G01.

[3] P. Castillo, A. Dzul and R. Lozano, "Real-time stabilization and tracking of a four rotor mini rotorcraft, European Control Conference ECC'03, Cambridge, EJ.K.1-4 September 2003.

[4] X. Deng and L. Schenato and S. S. Sastry, Model identification and attitude Control for a Miaomechanical Flying insect lacluding Thorax and Sensor Models”, ICRA 2003, Taipei, Taiwan, (2004), pp. 1152-1157.

[5] G. Cai, B. M. Chen, I. T. Peng, M. Dong and T. H. Lee, "Modeling and control system design for a UAV helicopter", In Proceedings of the 14th Mediterranean conference on control and automation, (2006), pp. 210-215. 
[6] Y. He, B. M. Chen and C. Wu, "Composite nonlinear control with state and measurement feedback for general multivariable systems with input saturation”, Systems and Control Letters, vol. 54, (2005), pp. 455-469.

[7] J. M. Roberts, P. Corke and G. Buskey, "Low-cost flight control system for a small autonomous helicopter", In Proceedings of the 2002 Australian conference on robotics and automation, (2002), pp. 546-551.

[8] P. Melin and O. Castillo, "A new method for adaptive model-based control of non-linear dynamic plants using a neuro-fuzzy-fractal approach”, Soft Computing Journal, vol. 5, no. 2, (2001), pp. 171-177.

[9] P. Melin and O. Castillo, "Intelligent control of complex electrochemical systems with a neuro-fuzzygenetic approach", IEEE Transactions on Industrial Electronics, vol. 48, no. 5, (2001), pp. 951-955.

[10] S. Nakamura, "Numerical Analysis and Graphic Visualization with MATLAB", Prentice-Hall, (1997).

[11] S. Bouabdallah and A. Noth, "Siegwart R.PID vs. LQ Control Techniques Applied to an Indoor MicroQuadrotor", IEEE International Conference on Intelligent Robots and Systems, (2004).

[12] M Krstic, I Kanellakopoulos and P Kokotovic, "Nonlinear and adaptive control design", John Wiley \& Sons, New York, (1995).

[13] P. A. Ioannou and J. Sun, "Robust adaptive control", Prentice Hall, Upper Saddle Press NJ, USA, (1996).

[14] C. H. Hong, K. C. Choi and B. S. Kim, "Applications of Adaptive Neural Network Control to an Unmanned Airship", International Journal of Control, Automation, and Systems, vol. 7, no. 6, (2009), pp. 911-917.

[15] P. McKerrow, "Modelling the Draganflyer four-rotor helicopter", in proceedings of the 2004 IEEE International Conference Robotics and Automation, (2004). 
International Journal of Multimedia and Ubiquitous Engineering Vol.12, No.1 (2017) 